\title{
Over-ground walking in Parkinson's disease: A pilot study utilizing a portable metabolic analyzer
}

\author{
Alyssa D. Stookey ${ }^{1}$, Frederick M. Ivey ${ }^{1,2}$, Jessica E. Hammers ${ }^{1}$, Lisa M. Shulman ${ }^{2}$, \\ Karen Anderson ${ }^{3}$, Leslie I. Katzel ${ }^{4,5^{*}}$ \\ ${ }^{1}$ VA Maryland Exercise and Robotics Center of Excellence, Baltimore, USA \\ ${ }^{2}$ Department of Neurology, University of Maryland School of Medicine, Baltimore, USA \\ ${ }^{3}$ Department of Psychiatry, University of Maryland School of Medicine, Baltimore, USA \\ ${ }^{4}$ Division of Gerontology and Geriatric Medicine, Department of Medicine, University of Maryland School of Medicine, Baltimore, \\ USA; *Corresponding Author: 1katzel@,grecc.umaryland.edu \\ ${ }^{5}$ Baltimore VA Medical Center, Geriatrics Research Education and Clinical Center (GRECC), Baltimore, USA
}

Received 2 October 2012; revised 5 November 2012; accepted 11 November 2012

\section{ABSTRACT}

Alterations in gait biomechanics are common during early stages of Parkinson's disease (PD), potentially elevating energy requirements of walking and leading to impaired economy of gait. Although gait economy is traditionally assessed during treadmill walking with simultaneous oxygen consumption $\left(\mathrm{VO}_{2}\right)$ monitoring, treadmill gait mechanics, particularly in PD, may be different from over-ground walking mechanics, possibly providing a distorted picture of true gait economy. Currently, no studies have directly examined the energy cost of over-ground walking in PD patients. The purpose of this study was to test the feasibility of measuring energy expenditure during over-ground walking in mild to moderate PD using portable gas exchange monitoring technology. Additionally, we sought to determine whether energy expenditure, as assessed through $\mathrm{VO}_{2}$ measures, related to disease severity for PD. Seventeen PD patients underwent separate 6-minute walk (6MW) tests both with and without the COSMED K4b2 portable oxygen monitoring system. Gait economy was calculated as measured $\mathrm{VO}_{2}$ during $6 \mathrm{MW}$ divided by the predicted $\mathrm{VO}_{2}$ for non-PD age-matched subjects, according to a standard estimation equation utilizing ground speed. Distance covered during the $6 \mathrm{MW}$ with the portable system (420 \pm 12 meters) was highly correlated $(r=0.96, p<0.001)$ to distance without the system ( $442 \pm 135$ meters) indicating that the lightweight equipment did not confound walking ability in these participants. Mean $\mathrm{VO}_{2}$ during the $6 \mathrm{MW}(16.0 \pm 5.5 \mathrm{~mL} / \mathrm{kg} / \mathrm{min})$ was $74 \%$ of mean $\mathrm{VO}_{2}$ peak for this sample, and 16 out of
17 subjects had impaired gait economy. The degree of gait economy impairment was not related to scores on either UPDRS total or motor. Our results demonstrate that PD patients use extraordinarily high percentage of $\mathrm{VO}_{2}$ peak for normal floor walking, and show impaired gait economy relative to prediction equations. Interestingly, the degree of elevated energy expenditure during gait did not relate to disease severity.

Keywords: Gait Economy; Oxygen Consumption; $\mathrm{K} 4 \mathrm{~b} 2$

\section{INTRODUCTION}

Gait impairment is one of the cardinal symptoms of Parkinson's Disease (PD). Alterations in the biomechanics of gait, such as decreased stride length, increased stride length variability and reduced gait speed, are common even in early-stage PD [1-3]. Most often, PD patients attempt to compensate for short steps by increasing gait cadence, thereby potentially elevating energy requirements of usual pace walking. Increased energy expenditure during walking, manifested as higher oxygen consumption $\left(\mathrm{VO}_{2}\right)$, reflects inefficient gait or lower economy of gait [4], with activities being performed at a higher percentage of an individual's maximal or peak aerobic capacity $\left(\mathrm{VO}_{2}\right.$ peak). The clinical significance is that impaired economy of gait and decreased physiological reserve often lead to increased fatigue and reduced endurance, thereby limiting functional independence and sustainability of activities of daily living (ADLs).

Measurement of gait economy using $\mathrm{VO}_{2}$ measurements is traditionally done during submaximal treadmill walking, but this technique is arguably confounded by a 
number of features, especially in neurologically disabled populations. Specifically, a moving treadmill belt alters gait mechanics to the extent that submaximal $\mathrm{VO}_{2}$ values during treadmill walking may not reflect the individual's true over-ground gait economy [5]. For this reason, methods of capturing economy of gait in more realistic over-ground settings must be considered and developed to accurately assess gait efficiency in PD. Recent advancements in portable $\mathrm{VO}_{2}$ measurement technologies have opened many possibilities that stationary metabolic equipment never afforded.

The 6-minute walk (6MW) is a test commonly used to assess longer distance ambulatory capacity over-ground. Hence, we chose to combine state-of-the-art portable metabolic monitoring equipment (COSMED USA; Chicago, IL) with 6MW testing for the first time in PD to directly examine the energy costs of over-ground walking (economy of gait) in mild to moderately severe PD patients. The specific aims of this pilot project were to test the feasibility of measuring submaximal energy expenditure $\left(\mathrm{VO}_{2}\right)$ during the $6 \mathrm{MW}$ in mild to moderate $\mathrm{PD}$ using the $\mathrm{K} 4 \mathrm{~b} 2$ portable oxygen monitoring system, and to determine whether submaximal energy expenditure related to commonly applied disease severity scales (UPDRS).

\section{METHODS}

\subsection{Subjects}

This study was approved by the Institutional Review Board at the University of Maryland Baltimore and written informed consent was obtained from each participant. Seventeen subjects with mild to moderately severe PD were recruited from the University of Maryland Parkinson's Disease and Movement Disorders Center, the Baltimore VA Medical Center Parkinson's Disease Clinic and via media advertisements. Each participant agreed to participate in a larger randomized trial (beyond the scope of this paper) examining the effects of exercise training on executive function in PD. During the initial history and physical examination, the Hoehn and Yahr (HY) scale [6] and Unified Parkinson's Disease Rating Scale (UPDRS) [7] were administered by a neurologist (LMS) with expertise in movement disorders. Study inclusion criteria were: 1) diagnosis of levodopa-responsive PD characterized by 2 of 3 cardinal signs (resting tremor, bradykinesia, rigidity; 2) HY stage 2 to 3 (while "on" for motor fluctuations); 3 ) presence of mild to moderate gait impairment, (score of 1 or 2 on the UPDRS questions \#29 Gait or \#30 Postural Stability); and 4) unlikely to require PD medication adjustment for 4 months. Study exclusion criteria included: 1) folstein mini mental status examination score [8] less than 23; 2) unstable cardiac, pulmonary, liver or renal disease; 3 ) poorly controlled hypertension or diabetes; 4) anemia, orthopedic or chronic pain condition restricting exercise; 5) severe depression or other unstable psychiatric condition; and 6) >20 minutes of aerobic exercise more than 3 times per week (to avoid prior training effect).

\subsection{Assessments}

All physical performance measures used in this substudy were performed on separate days to avoid the confounding effects of fatigue. Further, all study evaluations (rating scales and functional tests) were performed in the early afternoon while the subjects were "on" or within 3 hours of medication intake. By performing the tests at the same time of day we attempted to control for confounding effects of medication timing issues. Subjects used an additional dose of medication to maintain the "on" state for assessment when necessary.

\subsection{Exercise Treadmill Test with Measurement of $\mathrm{VO}_{2}$ Peak}

After an initial screening graded exercise test performed on a separate day to exclude subjects with previously undiagnosed silent ischemia, cardiac arrhythmias or severe exercise-induced hypotension, subjects underwent a progressively graded exercise treadmill test to voluntary exhaustion with measurement of $\mathrm{VO}_{2}$ peak using a stationary Quark Cardio Pulmonary Exercise Testing metabolic analyzer (COSMED, USA; Chicago, IL) as previously described [9]. Oxygen consumption, carbon dioxide production, and minute ventilation were measured breath-by-breath and values averaged for 20 second intervals. Subjects were instructed not to talk during the test as this affects the depth of breathing and gas exchange. The $\mathrm{VO}_{2}$ peak was based on the mean of the final two 20 second averages obtained during the final stage of the test. The results of this test were compared with submaximal over-ground measurements to ascertain what percentage of peak $\mathrm{VO}_{2}$ was required for usual floor walking.

\section{4. $6 \mathrm{MW}$}

On separate testing days, the $6 \mathrm{MW}$ tests were performed both with and without the COSMED K4b2 portable oxygen monitoring system. Subjects were instructed to cover as much distance as possible during the $6 \mathrm{~min}$ utes. Both tests were conducted down the same hospital corridor with subjects turning every 100 feet, as prompted by orange traffic cones. For safety reasons, given concerns that subjects might not tolerate walking with the portable metabolic system, and given their increased fall risk, we decided not to perform the two tests in a random order as the first uninstrumented test allowed us to ob- 
serve their "natural" gait and walking patterns and potentially exclude subjects who were unable to complete the walks. There were no adverse events during the walks, and the portable system was well tolerated, with all of the subjects completing both walks. The staff was blinded to the results of the first test.

\subsection{Portable Oxygen Monitoring}

The K4b2 portable system, weighing less than $1 \mathrm{~kg}$, was worn on the chest, consisting of a small battery pack and portable gas analyzer. Breath-by-breath analysis was obtained using a flexible rubber facemask with flowmeter that attached to the portable system. To enhance comfort and familiarity, participants underwent a period of standing acclimatization, with the portable gear in place, prior to performing the $6 \mathrm{MW}$ test. Additionally, before beginning the $6 \mathrm{MW}$ test, participants sat quietly for 5 minutes while wearing the portable system. Participants were instructed not to talk during the walk to avoid affecting depth of breathing and gas exchange measurements. Based on K4b2 portable monitoring data obtained during the final 3 minutes of the $6 \mathrm{MW}$ tests, we calculated mean $\mathrm{VO}_{2}$.

\subsection{Economy of Gait}

Economy of gait was calculated as the measured $\mathrm{VO}_{2}$ during the $6 \mathrm{MW}$ divided by the predicted $\mathrm{VO}_{2}$ for non-PD aged-matched subjects based on the commonly accepted American College of Sports Medicine equation for walking accounting for both speed and grade [10].

$$
\begin{aligned}
& \mathrm{VO}_{2}=\text { horizontal component } \\
& \quad+\text { vertical component + resting component } \\
& \mathrm{VO}_{2}(\mathrm{~mL} / \mathrm{kg} / \mathrm{min}) \\
& =0.1(\text { speed })+1.8(\text { speed })(\text { fractional grade })+3.5
\end{aligned}
$$

In the case of the over-ground 6MW test, there was obviously no vertical element or grade to consider, such that variability in the predicted level was entirely a function of floor waking speed. Therefore, with grade at $0 \%$ the equation for predicted $\mathrm{VO}_{2}$ at a given floor walking speed was reduced to:

$$
\mathrm{VO}_{2}=0.1(\text { speed })+3.5 \text {. }
$$

According to our method for assessing gait economy in this pilot study, if the $\mathrm{PD}$ participant's $\mathrm{VO}_{2}$ for a given walking speed was higher than the predicted $\mathrm{VO}_{2}$ for that gait speed, they were labeled as having impaired economy of gait.

\subsection{UPDRS}

UPDRS, a commonly applied index for disease sever- ity, was administered and scored by a neurologist (LMS) with expertise in movement disorders. The Total UPDRS includes three subscales: Mentation, Behavior and Mood (Part I), Activities of Daily Living (Part II), and the Motor Examination (Part III). Both the total score and motor scores were used to compare with over-ground gait economy.

\section{STATISTICAL ANALYSIS}

SPSS Predictive Analytics Software 18.0 (SPSS, Inc. Chicago, IL) was used for statistical analysis. Descriptive statistics are expressed as mean \pm standard deviation (SD). Pearson's correlation coefficients were used to quantify strength of relationships between variables. An independent samples t-test was performed to compare measured versus predicted $\mathrm{VO}_{2}$ during the $6 \mathrm{MW}$ and a paired samples t-test was performed to compare mean $6 \mathrm{MW}$ distance with and without the portable system. All statistical tests were two sided, with $\mathrm{P}$ value $<0.05$ required for significance.

\section{RESULTS}

A total of 17 subjects, 12 male and 5 female, with a mean age $66.5 \pm 9.5$ years (range 46 to 79 years) were included in the analyses for this study. The mean HY score, UPDRS total score, and UPDRS motor score were reflective of mild to moderate disability. These and other subject characteristics are summarized in Table 1.

The mean distance covered during the uninstrumented $6 \mathrm{MW}$ was $420 \pm 120$ meters and the distance covered with subjects wearing the portable oxygen system was virtually identical at $442 \pm 135$ meters, indicating that the monitoring equipment did not in any way alter the capacity

Table 1. Subject characteristics and severity of PD.

\begin{tabular}{ccc}
\hline & Mean \pm SD & Range \\
\hline Age (years) & $66.5 \pm 9.5$ & $46-79$ \\
UPDRS total score & $43.47 \pm 16.93$ & $23-86$ \\
UPDRS motor & $25.65 \pm 14.56$ & $9-69$ \\
HY stage & $2.44 \pm 0.30$ & $2.0-3.0$ \\
HY stage 2.0 & $n=4(24 \%)$ & - \\
HY stage 2.5 & $n=11(12 \%)$ & - \\
HY stage 3.0 & $n=2(12 \%)$ & - \\
6MW VO 2 (mL/kg/min) & $16.0 \pm 5.5$ & $10.2-29.3$ \\
Peak VO $(\mathrm{mL} / \mathrm{kg} / \mathrm{min})$ & $22.9 \pm 9.3$ & $12.6+45.8$ \\
Self-selected walking & $2.3 \pm 0.72$ & $0.80-3.6$ \\
speed (mph) & $421 \pm 135$ & $158-679$ \\
6MW distance (m) & &
\end{tabular}


to ambulate over-ground. There was no significant difference in distance walked between the two $6 \mathrm{MW}$ tests $(\mathrm{p}=0.88)$. Furthermore, the distance covered between the 2 tests was highly correlated $(\mathrm{r}=0.96, \mathrm{p}<0.001$, Figure 1), and the portable $\mathrm{VO}_{2}$ system was well tolerated, with no falls or adverse events during testing.

Mean $\mathrm{VO}_{2}$ during the $6 \mathrm{MW}$ was $16.0 \pm 5.5 \mathrm{~mL} / \mathrm{kg} / \mathrm{min}$, which represented $74 \% \pm 21 \%$ of the mean $\mathrm{VO}_{2}$ peak for this sample. In three of the subjects, the $\mathrm{VO}_{2}$ measured during the $6 \mathrm{MW}$ exceeded the values obtained during their peak effort treadmill test. There was a significant difference between mean measured submaximal $\mathrm{VO}_{2}$ and mean predicted $\mathrm{VO}_{2}(10.5 \pm 2.3 \mathrm{~mL} / \mathrm{kg} / \mathrm{min})(\mathrm{p}<0.001)$, with measured values significantly higher than predicted ( $p<0.001$; Figure 2). In 16 of 17 subjects, the measured $\mathrm{VO}_{2}$ values during the $6 \mathrm{MW}$ exceeded the predicted values for non-PD subjects based on gait velocity (Figure 2), consistent with impaired economy of gait. On average, our subjects utilized 53\% more oxygen than expected (Figure 3).

Our chosen gait economy index (measured $\mathrm{VO}_{2}$ during $6 \mathrm{MW}$ divided by predicted $\mathrm{VO}_{2}$ according to gait speed) was not related to either UPDRS total $(r=-0.093)$ or motor $(\mathrm{r}=0.087)$ scale scores. Mean submaximal $\mathrm{VO}_{2}$ was also not related to UPDRS total $(\mathrm{r}=-0.13)$ or motor $(\mathrm{r}=-0.33)$. However, mean $\mathrm{VO}_{2}$ during the $6 \mathrm{MW}$ correlated with walking speed $(\mathrm{r}=0.67)$. The distance covered during the $6 \mathrm{MW}$ was negatively correlated with UPDRS motor $(\mathrm{r}=-0.5)$, but not UPDRS total.

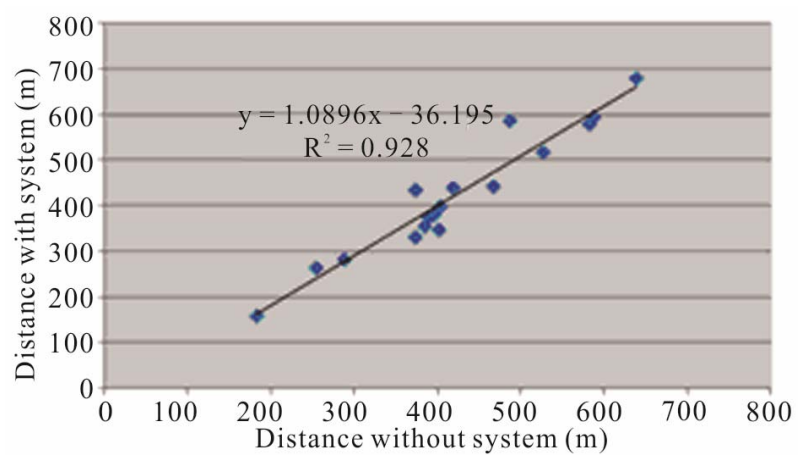

Figure 1. Distance covered without and with portable system.

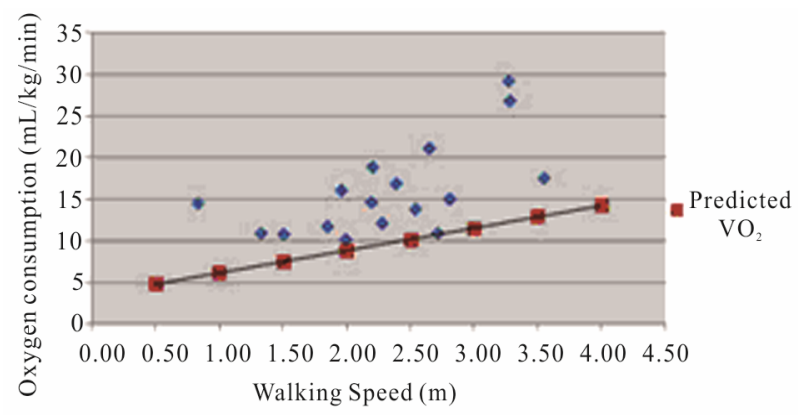

Figure 2. Measured submaximal $\mathrm{VO}_{2}$ versus predicted.



Figure 3. Average predicted vs measured submaximal $\mathrm{VO}_{2}$.

\section{DISCUSSION}

This study was the first to use novel, state-of-the art portable technology to directly examine the energy cost of over-ground walking in mild to moderate PD. Importantly, our study demonstrated the feasibility of using portable oxygen monitoring during over-ground walking in this population. According to our data, the K4b2 portable device was lightweight enough so as not to interfere with walking performances as evidenced by the identical $6 \mathrm{MW}$ distances between test one and test two. Therefore, this portable technology appears to be a suitable tool for use in this population. Our results are also the first to demonstrate the extent of over-ground gait economy impairment in PD and that over-ground energy expenditure is not predicted by commonly applied disease severity scales.

Few studies have directly measured walking economy based on submaximal oxygen consumption measures in PD. Of these, all assessed economy of gait during treadmill walking $[9,11]$, which, as mentioned previously, is confounded by several features. Christiansen, et al. [11] examined gait economy at various treadmill walking speeds in subjects with PD compared to healthy subjects without PD. They reported that submaximal $\mathrm{VO}_{2}$ was 6 to $10 \%$ higher in people with $\mathrm{PD}$ at various walking speeds above $1 \mathrm{mph}$. This stands in contrast to our current results, which suggest a much higher $\mathrm{VO}_{2}$ than predicted for a given rate of speed. In fact, our participants were consuming 53\% more oxygen than expected during the over-ground 6-minute walk test. Similarly, Katzel et al. [9] measured submaximal $\mathrm{VO}_{2}$ during treadmill walking at self-selected speed and showed a much greater impairment in walking economy. In that submaximal treadmill study, PD participants were operating at $64 \%$ of their measured $\mathrm{VO}_{2}$ peak, whereas the over-ground walking in this study required a mean $74 \%$ of $\mathrm{VO}_{2}$ peak. This 
$10 \%$ higher fractional utilization during floor walking supports our hypothesis that over-ground ambulation is more taxing and perhaps more reflective of true gait economy absent the artificial constraints of a treadmill testing environment. In seniors without neurologic disability, fractional utilization rates of $32 \%, 42 \%$, and $50 \%$ were reported for low, moderate, and high submaximal workloads, respectively [12], which is far more efficient than the rates observed in the current study. Additionally, our submaximal $\mathrm{VO}_{2}$ levels during floor walking in mild-moderate PD were 34\% higher than reported in healthy, elderly subjects when walking at their fastest comfortable speed on a treadmill [12]. In short, the PD participants in the current study had submaximal walking $\mathrm{VO}_{2}$ levels reflective of severe impairments in gait economy.

Our cohort of $17 \mathrm{PD}$ subjects had an average $\mathrm{VO}_{2}$ peak of $22.9 \pm 9.3 \mathrm{~mL} / \mathrm{kg} / \mathrm{min}$, which is consistent with values reported in the literature for this population $[9,13]$. Previous reports indicate that mild to moderate PD patients have $\mathrm{VO}_{2}$ peak values that are $20 \%-25 \%$ lower than healthy age-matched controls $[13,14]$. An even more discouraging picture emerges when this impairment in $\mathrm{VO}_{2}$ peak is coupled with lower gait economy during over-ground walking in $\mathrm{PD}$, perhaps explaining the inability of this population to sustain normal ADLs for any extended period of time. The 6MW distances observed in this study for PD were quite a bit lower than those previously reported in non-PD studies, supporting the idea that higher than normal submaximal $\mathrm{VO}_{2}$ levels translate into clinically relevant functional limitations. Our PD subjects' 6MW distances were comparable to the values reported in the existing literature for mild to moderate PD $[9,15]$ and were $18 \%$ lower than that predicted for healthy, non-PD individuals according to an established prediction equation, which takes into account age, gender, and weight [16]. This is consistent with Katzel, et al. [9] who reported a $17 \%$ difference.

Researchers have hypothesized that tremor, prominent at rest in people with PD, may play a role in economy of gait for individuals in early stages of PD due to the potential effect on resting energy expenditure. However, Christiansen, et al. [11] reported no significant difference in resting energy expenditure between PD patients $(3.5 \pm$ $0.7 \mathrm{~mL} / \mathrm{kg} / \mathrm{min})$ and healthy individuals $(3.5 \pm 0.5$ $\mathrm{mL} / \mathrm{kg} / \mathrm{min})(\mathrm{p}=0.97)$. Thus, it is reasonable to conclude that elevations in energy expenditure during submaximal walking are not a function of elevated resting $\mathrm{VO}_{2}$. Our data appears similarly unaffected by resting $\mathrm{VO}_{2}$ given an average resting level of $3.80 \pm 0.98 \mathrm{~mL} / \mathrm{kg} / \mathrm{min}$ in the 17 subjects studied.

Our results showed that economy of gait during over-ground walking was not related to PD severity. This is also consistent with previous research demonstrating no relationship between UPDRS total or motor and economy of gait during treadmill walking $[11,13]$. Christiansen, et al. [11], reported no relationship between submaximal $\mathrm{VO}_{2}$ and UPDRS total during submaximal treadmill walking, which combined with our over-ground findings call into question the utility of the UPDRS for predicting this particular aspect of post PD function. A lack of relationship between UPDRS and these measures may partly be a consequence of under-emphasis on ambulatory function and associated elements of endurance by this severity scale.

Our pilot results should be interpreted with caution based upon a number of clear limitations. First, our study determined economy of gait based upon a commonly accepted predicted $\mathrm{VO}_{2}$ equation instead of actually measuring $\mathrm{VO}_{2}$ in age and gender matched non-PD, healthy controls. However, it would have been difficult to find healthy subjects who walk slowly enough to match our PD subjects since slower walking speeds typically indicate functional and mobility impairments. Furthermore, this prediction equation is derived from treadmill walking, which qualifies as a potential limitation. During 6MW testing, subjects had to make tight turns around a cone every 100 feet, which could have had an adverse impact on distance covered and elicited a higher energy expenditure in subjects with limited ability to turn and/or balance impairments. Due to our small sample size, we were unable to analyze potential cofounders, such as height and how this may have affected stride length, and subsequently economy of gait. Stride length is known to influence walking economy in healthy individuals [17]. Lastly, our subjects were limited in range of $\mathrm{PD}$ severity (mild to moderate) due to entry criteria, calling into question the generalizability of our findings. Additionally, the lack of a relationship between disease severity and economy of over-ground gait reported in our study may have been attributed to this small range of disease severity.

\section{CONCLUSION}

We conclude that PD causes major impairments in over-ground gait economy, with clinical consequences that include lower functional capacity. Based on the established feasibility of such over-ground measures demonstrated in this study, future clinicians and researchers may wish to utilize these assessments in a number of ways. Direct measures of over-ground gait economy could serve as an index for determining response to therapy, exercise interventions, and/or progression of disease. Further, simultaneous measurements of gait biomechanics during floor walking could yield clues about the gait therapies that have the greatest potential for reversing the decrements in gait economy that accompany PD. 


\section{ACKNOWLEDGEMENTS}

This study was supported by VA RR\&D Career Development Award1 (Stookey), VA Merit Award funding (Ivey, Katzel, Anderson), Baltimore VA Medical Center Geriatric Research, Education and Clinical Center (GRECC), National Institute on Aging (NIA), Claude D. Pepper Older Americans Independence Center (P30-AG028747) and the Department of Veterans Affairs VA RR\&D Exercise \& Robotics Center of Excellence.

\section{REFERENCES}

[1] Brusse, K.J., Zimdars, S., Zalewski, K.R. and Steffen, T.M. (2005) Testing functional performance in people with Parkinson disease. Physical Therapy, 85, 134-141.

[2] Hausdorff, J.M. (2009) Gait dynamics in Parkinson's disease: Common and distinct behavior among stride length, gait variability, and fractal-like scaling. Chaos, 19, 1-14. doi:10.1063/1.3147408

[3] Bloem, B.R., Hausdorff, J.M., Visser, J.E. and Giladi, N. (2004) Falls and freezing of Gait in Parkinson's disease: A review of two interconnected, episodic phenomena. Movement Disorders, 19, 871-884. doi:10.1002/mds.20115

[4] Waters, R.L. and Mulroy S. (1999). The energy expenditure of normal and pathological gait. Gait Posture, 9, 207231. doi:10.1016/S0966-6362(99)00009-0

[5] Frenkel-Toledo, S., Giladi, N., Peretz, C., Herman, T., Gruendlinger, L. and Hausdorff, J.M. (2005) Treadmill walking as an external pacemaker to improve gait rhythm and stability in Parkinson's disease. Movement Disorders, 20, 1109-1114. doi:10.1002/mds.20507

[6] Hoehn, M.M. and Yahr, M.D. (1967) Parkisonism: Onset, progression and mortality. Neurology, 17, 427-442. doi:10.1212/WNL.17.5.427

[7] Fahn, S., Elton, R.L. and Committee, U.D. (1987) Unified Parkinson's disease rating scale. In: Fahn, S., Marsden, C.D., Calne, D. and Goldstein, M., Eds., Recent Developments in Parkinson's Disease. Macmillan Healthcare Information, Florham Park, New York, 153-163.

[8] Folstein, M.F., Folstein, S.E. and McHugh, P.R. (1975)
Mini mental state: A practical method for grading the cognitive state of patients for the clinician. Journal of Psychiatric Research, 12, 189-198. doi:10.1016/0022-3956(75)90026-6

[9] Katzel, L.I., Ivey, F.M., Sorkin, J.D., Macko, R.F., Smith, B. and Shulman, L.M. (2012) Impaired economy of gait and decreased six-minute walk distance in Parkinson's disease. Parkinson's Disease, 241754.

[10] Whaley, M.H., Brubaker, P.H. and Otoo, R.M. (2006) Metabolic calculations, in ACSM's Guidelines for Exercise Testing and Prescription. 7th Edition, Lipinncott Williams and Wilkins, Philadelphia.

[11] Christiansen, C.L., Schenkman, M.L., McFann, K., Wolfe, P. and Kohrt, W.M. (2009) Walking economy in people with Parkinson's disease. Movement Disorders, 30, 14811487. doi:10.1002/mds. 22621

[12] Arnett, S.W., Laity, J.H., Agrawal, S.K. and Cress, M.E. (2008) Aerobic reserve and physical functional performance in older adults. Age and Ageing, 37, 384-389. doi:10.1093/ageing/afn022

[13] Kazel, L.I., Sorkin, J.D., Macko, R.F., Smith, B., Ivey, F.M. and Shulman, L.M. (2011) Repeatability of aerobic capacity measurements in Parkinson Disease. Medicine \& Science in Sports \& Exercise, 43, 2381-2387. doi:10.1249/MSS.0b013e31822432d4

[14] Rosen, M.J., Sorkin, J.D., Goldberg, A.P., Hagberg, J.M. and Katzel, L.I. (1998) Predictors of age-associated decline in maximal aerobic capacity: A comparison of four statistical models. Journal of Applied Physiology, 84, 2163-2170.

[15] Falvo, M.J. and Earhart, G.M. (2009) Reference equation for 6-minute walk in individuals with Parkinson disease. Journal of Rehabilitation Research and Development, 46, 1121-1126. doi:10.1682/JRRD.2009.04.0046

[16] Enrichi, P.L. and Sherrill, D.L. (1998) Reference equation for the six-minute walk in healthy adults. American Journal of Respiratory and Critical Care Medicine, 158, 1384-1387.

[17] Martin, P.E. and Morgan, D.W. (1992) Biomechanical considerations for economical walking and running. Medicine \& Science in Sports \& Exercise, 24, 467-474. doi:10.1249/00005768-199204000-00013 